\title{
Compact and lightweight 3D printed platform for testing attitude determination and control system of small satellites
}

\author{
J Bryła1, P Zagorski², D Knapik², P Sleczka', \\ P Zdziebko', A Gallina' \\ 1. Faculty of Mechanical Engineering and Robotics, $\mathrm{AGH}$ \\ University of Science and Technology, Kraków, Poland \\ 2. Faculty of Electrical Engineering Computer Science and \\ Biomedical Engineering, AGH University of Science and \\ Technology, Kraków, Poland
}

\begin{abstract}
Ground tests of the attitude control system of small satellites can be performed with a test stand that simulates torque free rotations. It consists of a floating platform mounted on a spherical frictionless air bearing joint and equipped with a balancing system that removes the gravity torque acting upon the system. An important design requirement of such platforms is its reduced weight and inertia. In this work we propose an original lightweight 3D printed floating platform made of polylactide (PLA). The utilization of plastic for structural elements is a novelty for this kind of devices, but it also introduces some challenging aspects. Indeed, the reduced stiffness of PLA, when compared with aluminum commonly used in these applications, negatively affects the balancing process. Nonetheless, thanks to the peculiar design we adopted, the platform shows high performance in terms of structural rigidity and balancing accuracy. Experiments evidence that the platform is able to reduce gravity torques to a few tens of micro-Newton meters and a residual offset between the center of mass and center of rotation of about 10 micro meters. These values agree with those of solutions presented in the literature.
\end{abstract}

\section{INTRODUCTION}

The 2020 was a record-breaking year in terms of number of small satellites launches, with as many as 1163 spacecrafts sent into orbit. And the 2021 seems to continue this trend with already 757 satellite deployments only in the first quarter [1]. The majority of launches of this year were Starlink satellites: 528 new spacecrafts with mass of about $260 \mathrm{~kg}$ put into orbit. Almost all remining launches were nanosatellites and picosatellites, with weight below $10 \mathrm{~kg}$. Also, future programs foresee small satellite clusters or constellations conducting advanced tasks. The decrease of the satellite size seems also a trend for the years to come. The miniaturization of spacecraft components has made small satellites technically advanced. Nowadays, almost all nanosatellites include a more or less sophisticated attitude determination and control system (ADCS).

$\overline{{ }^{*} \text { Corresponding Author: agallina@agh.edu.pl }}$ 
The performance and reliability of this important component is commonly tested on the ground by means of air bearing test stands composed of a floating platform mounted on a spherical air bearing. This facility allows one to simulate torque-free conditions for rotations similar to those experienced by a spacecraft in orbit. Examples of realizations of this facility are presented in [2-5]. An important design requirement of these systems is related to their mass and inertia. Since the floating platform adds mass and inertia to the article under testing, it is important to keep these values low enough such to not alter the test article performance assessment. This is true especially when testing ADCS of small satellites with limited control authority [6-8]. On the other hand, a floating platform has to embed a balancing system that removes gravitational torques by controlling the position of small counterweights. Thus, the design of a lightweight and compact platform becomes a challenging task.

In this paper we present a solution that makes use of 3D printed PLA elements instead of commonly used aluminum components. To the best of our knowledge, the utilization of FDM technology in systems with high balancing precision, as the one we are presenting, is new. However, since the platform stiffness is a fundamental parameter for achieving accurate balancing, then the effect of structural deformation due to the usage of PLA is studied in depth. Experiments show that the performance of our solution is comparable with those of best-inclass solutions, although using an economic and simple manufacturing process [9]. This is very convenient, especially in academic satellite projects with limited budget.

The article is structured as follows. In Section 2 we describe mechanical and electronic aspects of the platform design. In Section 3 we focus on the algorithm used to balance the system. Results from experiments are exhibited in Section 4 and conclusions provided in Section 5 .

\section{PLATFORM DESIGN}

The tabletop air bearing system developed in the AGH Space Tech Lab [10] uses a small bearing manufactured by Physik Instrumente with maximum payload of $15 \mathrm{~kg}$. The floating system is composed of three balancing arms evenly spaced around a flat circular plate. Each arm hosts a counterweight (CW) of $125 \mathrm{~g}$, which can slide along a rectilinear rail, and it is actuated by precise NEMA8 stepper motor manufactured by Haydon Kerk Pittman. The motion is transferred to the counterweights via a lead screw of $0.3048 \mathrm{~mm}$ thread. The angle between the arms and the table, in this work set to 126 deg, can be changed by substituting the arm-plate connectors. The tilted arm configuration of the system reduces the platform inertia with respect to a configuration where the CW paths are along the pitch, roll, yaw axes. The system is able to balance a test article mounted at the top of the plate of mass between about $500 \mathrm{~g}$ and $1200 \mathrm{~g}$ (when assuming that its center of mass is $40 \mathrm{~mm}$ above the plane of the plate). Several concepts, such as reliability and vulnerability, can be taken into account to manage these risks in desired systems. If larger payloads need to be tested, then additional weights can be fixed at the free extremes of the arms. The stiffness of the system is increased by a ring connecting the arms together. All parts are made of PLA except for the linear drivers, motors and the counterweight masses that are made of stainless steel. In the tested configuration the weight of the platform excluding counterweights is $915 \mathrm{~g}$ and that of the test article 615 g. A graphical description of all components is given in Fig. 1. Tab. 1 lists the inertia properties of the platform calculated from the $\mathrm{CAD}$ model with respect to the reference body frame depicted in Fig.1. 

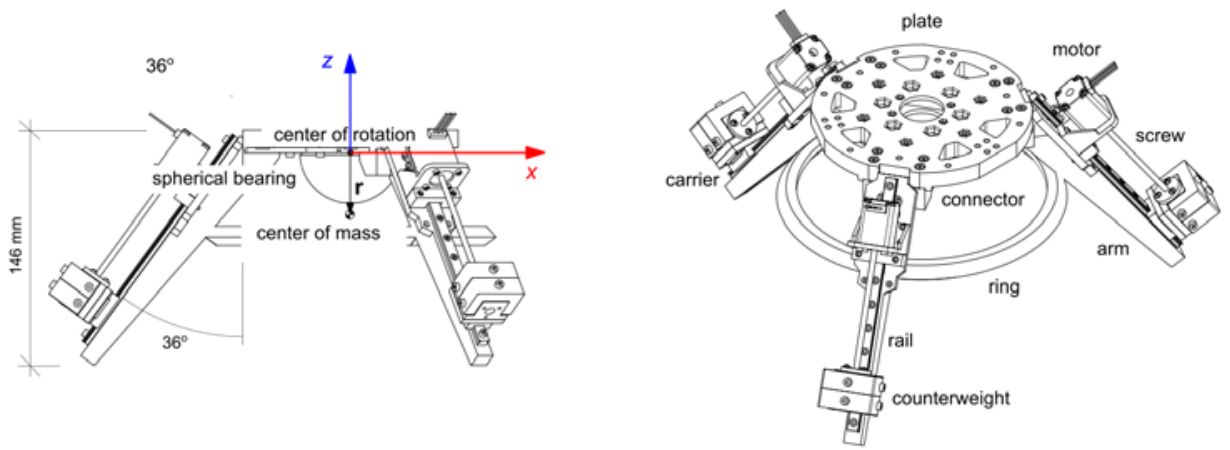

Figure 1. Views of the CAD model of the platform with its components. The colored frame plotted in the figure is the body reference frame used in this work. Its origin is at the center of rotation of the platform.

Table 1 - Moments of inertia of the platform for the two configurations when the three counterweights occupy their upper and lower position.

\begin{tabular}{llll}
\hline Counterweight position & $\boldsymbol{I}_{\boldsymbol{x} \boldsymbol{x}}[\mathbf{k g m m 2}]$ & $\boldsymbol{I}_{\boldsymbol{y} \boldsymbol{y}}[\mathbf{k g m m 2}]$ & $\boldsymbol{I}_{\boldsymbol{z Z}}$ [kgmm2] \\
\hline Top position & 11300 & 11460 & 14016 \\
Bottom position & 16550 & 16700 & 19020 \\
\hline
\end{tabular}

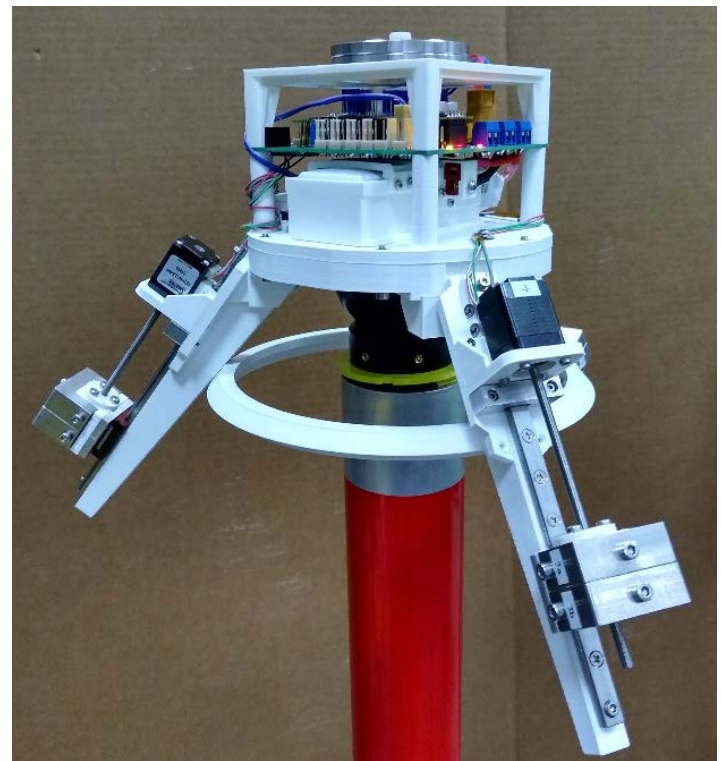

Figure 2. Physical model of the floating platform with the balancing systems avionics. 
The balancing system is controlled by embedded electronics whose heart is a STM32F446RET (Cortex-M based) microcontroller running the FreeRTOS real-time operating system. It collects measurements from the MPU9250 IMU sensor and communicates with the three TMC2209 drivers that control the stepper motors. The system possesses wireless communication capabilities by a Bluetooth module. The task priority system is used to ensure a deterministic data acquisition frequency of $100 \mathrm{~Hz}$ and to control the functions by the microcontroller. The module can also control reaction wheels and magnetorquers and to interface with sun sensors, features that are not used in this work. A picture of the platform with its avionics (appearing as the payload) is depicted in Fig. 2.

It is known that gravity forces acting upon the system produce structural deformations that may significantly affect the balancing accuracy [11]. Floating simulators are commonly made of aluminum, which is a stiffer material than PLA. Thus, the use of PLA implies larger structural deformations. In order to assess these latter and mitigate their consequences we performed FE analyses. The FE model is created in Altair HyperMesh and computed with MSC.Marc solver. The balancing masses and motors are modeled as concentrated masses. A linear quasi-static analysis is carried out for different tilted configurations of the platform and with the counterweights fixed slightly below their mid-way position on the rail. A top view of the platform FE model is given in Fig. 3. The analyzed configurations are those attained by rotating the platform of $20^{\circ}$ and $40^{\circ}$ about an axis of rotation laying on the horizontal xyplane. The axis of rotation is set at 5 different angle values, as also shown in Fig. 3. Altogether, 11 different platform poses are examined, including the configuration when the platform is horizontal. They evenly span the whole set of possible attitude configurations of the platform in its motion on the bearing. The structural deformations and the corresponding position of the center of mass of the platform were numerically calculated for the 11 configurations. The outcomes are presented in Tab. 2. The last column of the table gives the magnitude of the vector difference between the actual center of mass (CM) position vector and that of the reference configurations. The reference configuration is the one when the platform is horizontal (configuration nr 1 in Tab. 2). 


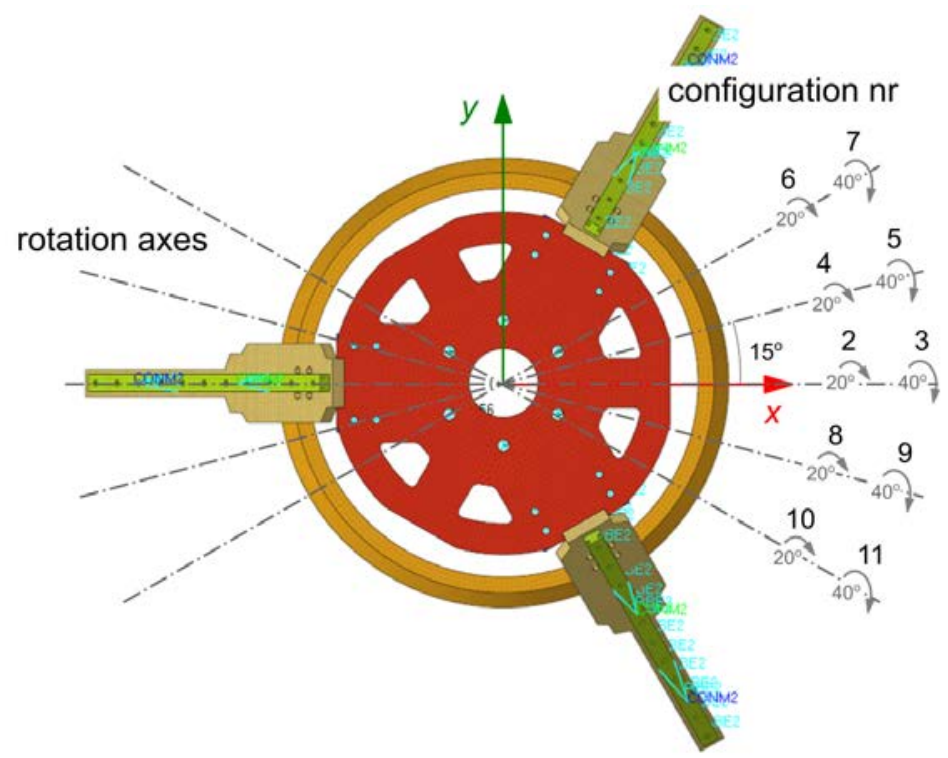

Figure 3. FE model of the platform used in the structural analysis. The examined platform attitudes are those resulting from tilting the platform about different rotation axes of $20^{\circ}$ and $40^{\circ}$. The rotation axes assumed are represented in the figure as dashed lines.

Table 2 - Positions for center of mass of the platform for different poses obtained from the FE analysis. The last column calculates the magnitude of the center of mass offset from the reference horizontal configuration (highlighted solution $\mathrm{nr} 1$ ).

\begin{tabular}{lllllll}
\hline $\mathbf{N r}$ & Axis angle $\left[{ }^{\circ}{ }^{\circ}\right.$ & Tilt angle $\left[^{\circ}\right]$ & $\boldsymbol{r}_{\boldsymbol{x}}[\mathbf{m m}]$ & $\boldsymbol{r}_{\boldsymbol{y}}[\mathbf{m m}]$ & $\boldsymbol{r}_{\boldsymbol{z}}[\mathbf{m m}]$ & $|\Delta \mathbf{r}|$ \\
\hline 1 & 0 & 0 & 0.0027 & 0.0053 & -36.9774 & - \\
2 & 0 & 20 & 0.0024 & 0.0007 & -36.9764 & 0.0047 \\
3 & 0 & 40 & 0.0026 & -0.0040 & -36.9736 & 0.0048 \\
4 & 15 & 20 & 0.0037 & 0.0006 & -36.9764 & 0.0046 \\
5 & 15 & 40 & 0.0047 & -0.0039 & -36.9737 & 0.0048 \\
6 & 30 & 20 & 0.0047 & 0.0012 & -36.9765 & 0.0047 \\
7 & 30 & 40 & 0.0069 & -0.0033 & -36.9738 & 0.0100 \\
8 & -15 & 20 & 0.0013 & 0.0008 & -36.9763 & 0.0100 \\
9 & -15 & 40 & 0.0004 & -0.0034 & -36.9738 & 0.0102 \\
10 & -30 & 20 & 0.0003 & 0.0014 & -36.9763 & 0.0097 \\
11 & -30 & 40 & -0.0017 & -0.0023 & -36.9737 & 0.0095 \\
\hline
\end{tabular}




\section{BALANCING ALGORITHM}

To balance the floating system, we can use either a manual or an automatic procedure. The former approach can be very precise, but also time consuming. When operating the system small changes of the payload assembly (e.g., cable movements, screw changes, etc.) can unbalance the system and call for a new balancing process. Hence, the process is usually automated by means of balancing algorithms. Two types of algorithms have been proposed in the literature: feedback and batch-based methods. Feedback methods are claimed to yield most accurate results $[1,12]$. However, they also require very precise sensors and are generally more difficult to implement [13]. Differently, offline batch-based methods [14, 15] show slightly lower performance, but are less demanding from the hardware point of view. In this work we adopt a batch-based method for automatic balancing. The position of the CM is calculated by the algorithm from onboard angular rate and accelerometer measurements by solving an inverse problem. The dynamic model employed in the solution is the well-known Euler equation

$$
\mathbf{J} \dot{\omega}+\boldsymbol{\omega} \times(\mathbf{J} \omega)=\mathbf{t}_{\mathrm{ext}}
$$

where $\mathbf{J}$ is the matrix of inertia of the system, $\boldsymbol{\omega}$ its angular velocity and $\mathbf{t}_{\text {ext }}$ the net external torque. For our floating system this torque is essentially due to gravity and aerodynamic effects. The aerodynamic effects can be neglected as they do not significantly affect the estimates when only the CM is estimated. The gravitational torque is

$$
\mathbf{t}_{\text {gravity }}=\mathbf{r} \times m \mathbf{g}
$$

with $\mathbf{r}$ the position vector of the center of mass of the system with respect to the center of mass (CR), $\mathrm{m}$ the mass of the system and $\mathbf{g}$ the vector of the gravitational acceleration. All vector components are expressed into the body frame depicted in Fig. 1. We can plug Eq. 2 into Eq. 1, which gives

$$
\mathbf{J} \dot{\boldsymbol{\omega}}+\boldsymbol{\omega} \times(\mathbf{J} \boldsymbol{\omega})=\mathbf{r} \times m \mathbf{g}
$$

We can rewrite the equation above in the matrix form,

$$
\mathbf{A r}=\mathbf{b}
$$

where

$$
A=m \mathbf{g}^{\times} \quad \text { and } \quad \mathbf{b}=\mathbf{J} \dot{\boldsymbol{\omega}}+\boldsymbol{\omega} \times(\mathbf{J} \boldsymbol{\omega})
$$




$$
\mathbf{g}^{\times}=\left(\begin{array}{ccc}
0 & -g_{z} & g_{y} \\
g_{z} & 0 & -g_{x} \\
-g_{y} & g_{x} & 0
\end{array}\right)
$$

Eq. 4 gives an explicit solution for $\mathrm{r}$ because all terms of $\mathrm{A}$ and $\mathrm{b}$ are known from measurements. However, an integral form is advocated because b depends on the angular acceleration $\omega$; which is subjected to large errors when calculated from a noisy $\omega$ signal. Thus, after integrating, Eq. 4 becomes

$$
\mathbf{C r}=\mathbf{d}
$$

where,

$$
\mathrm{C}=\int m \mathbf{g}^{\times} d t \quad \text { and } \quad \mathrm{d}=\int \mathrm{J} \dot{\omega}+\boldsymbol{\omega} \times(\mathrm{J} \omega) d t
$$

To regularize the solution a significantly large batch of measurements need to be used. In our work, we use timeseries of $50 \mathrm{~s}$ at a sampling frequency of $100 \mathrm{~Hz}$. It results in a $\boldsymbol{d}$ vector with about 15000 elements (5000 samples x 3 components). The solution of Eq. 7 is obtained by least squares method. Once $r$ is estimated, then the CW positions that make CM to be coincident to CT are calculated from the known geometry of the platform and afterwards implemented by the actuators.

\section{EXPERIMENTAL ANALYSIS}

We tested the performance of the platform by both manual and automated balancing. Manual balancing is executed by initially setting the CWs sensibly below their balancing positions and then progressively moving them upward, while ensuring that the platform stays horizontal. Thus, the gravitational torque is progressively reduced. This fact is evidenced by the increase of the oscillation period of the platform, if perturbed from the steady configuration. The manual process ends when any further upward movement the CWs makes the platform unstable (i.e. behaving like an inverted pendulum). In practice, with manual balancing we can reach the pendulum stable configuration of the system which is the closest one to that of exact balancing. Differently, automatic balancing is performed by setting the system with a pendulum stable configuration in motion by manual excitation. While the platform is tumbling, the onboard computer acquires measurements from the IMU sensor and transmits them to the ground station. After $50 \mathrm{~s}$ the acquisition is interrupted and received data are elaborated by the ground computer where the balancing algorithm is implemented. During the tests we noted that the accuracy of the CM estimate is dependent on the CW positions adopted in the experiment, as also reported in [14]. We also observed that the platform angular velocity affects the estimates. Thus, we decided to perform several experiments with different values of those parameters. The results are provided in Tab. 3 . 
Table 3 - Kinematic setup of the balancing experiments and corresponding estimates of the CM of the system without the CWs.

\begin{tabular}{lllllll}
\hline $\mathbf{E x p ~ N r}$ & $\underline{\boldsymbol{\omega}}[\mathbf{r a d} / \mathbf{s}]$ & $\boldsymbol{\Delta} \mathbf{s}[\mathbf{m m}]$ & $\boldsymbol{r}_{\boldsymbol{p} \boldsymbol{x}}[\mathbf{m m}]$ & $\boldsymbol{r}_{\boldsymbol{p} \boldsymbol{y}}[\mathbf{m m}]$ & $\boldsymbol{r}_{\boldsymbol{p} \boldsymbol{z}}[\mathbf{m m}]$ & $\left|\boldsymbol{\varepsilon}_{\mathbf{P}}\right|[\mathbf{m m}]$ \\
\hline 1 & 1.40 & -600 & 0.274 & 0.219 & 11.991 & 0.076 \\
2 & 1.19 & -600 & 0.274 & 0.220 & 12.000 & 0.085 \\
3 & 0.98 & -600 & 0.274 & 0.219 & 12.997 & 0.081 \\
4 & 2.65 & -450 & 0.272 & 0.215 & 11.958 & 0.043 \\
5 & 1.77 & -450 & 0.274 & 0.214 & 11.956 & 0.040 \\
6 & 0.93 & -450 & 0.246 & 0.213 & 11.952 & 0.037 \\
7 & 2.06 & -300 & 0.275 & 0.208 & 11.919 & 0.007 \\
8 & 1.63 & -300 & 0.276 & 0.207 & 11.916 & 0.005 \\
9 & 1.37 & -300 & 0.277 & 0.206 & 11.914 & 0.005 \\
10 (HAND) & - & - & 0.278 & 0.202 & 11.917 & - \\
\hline
\end{tabular}

In the table, the column headers $\underline{\omega}, \Delta \mathrm{s}, r_{p x}, r_{p y}$ and $r_{p z}$ denote the mean value of the platform angular speed during the experiment, the distance of the CWs from their top position and the three rectangular components of the estimated CM of the system, respectively. These components refer to the system without the CWs and are therefore confrontable. The last row of the table exhibits the CM position vector calculated from manual balancing, which can be considered as the reference value. Thus, the estimate accuracy can be assessed as the magnitude of the vector subtracting the CM estimates to the reference values. This metric is denoted by $\left|\varepsilon_{\mathrm{P}}\right|$ and it is presented in the last column of Tab. 3. A global metric assessing the algorithm balancing accuracy can be defined as the deviation of the estimated CM-CR offsets of the entire floating system (including CWs) from the same quantity obtained from manual balancing. The magnitude of these deviations is denoted by $\left|\varepsilon_{S}\right|$ and presented in Fig. 4. Inhere, the marker shape describes the position of the CWs in the experiment, while the marker color the average angular speed of the platform.

The diagram shows that performing an experiment with smaller platform speeds generally improves the estimates. Most of all, the accuracy is dependent on the location of the CWs and growing when getting closer to the balancing positions. The results in Fig. 4 show that in the third set of experiments the balancing algorithm is able to achieve an accuracy below 0.005 $\mathrm{mm}$. In practice, we think that the real accuracy is lower because limited by structural deformations. 


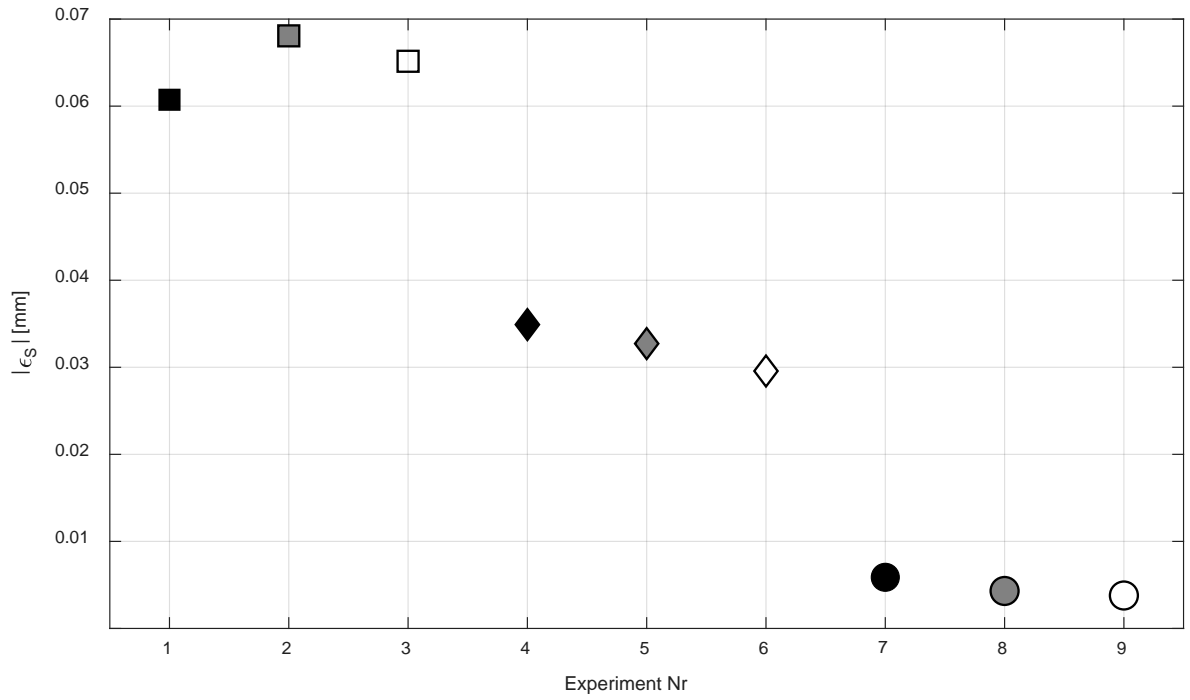

Figure 4. Magnitude of the automatic balancing error when compared with manual balancing for different experimental setups. The marker symbol denotes the CWs position of the particular experiment, the color the average angular speed of the platform in the experiment. Best CM estimates are when the CWs are close to their balancing configurations, resulting $\left|\varepsilon_{S}\right|$ below $0.005 \mathrm{~mm}$.

We already introduced this problem in Section 2 and examined it with numerical simulations. In this section we intend to investigate the issue experimentally by the analysis of residual moments. The residual CM-CR offset left after the balancing process generates a small torque acting upon the system. This torque, called residual torque, can be calculated from IMU measurements from Eq. 1. The magnitude of this torque we obtained after manually balancing is exhibited in the top diagram of Fig. 5. In the same figure the platform tilt angle obtained from IMU measurements is also presented. From these two diagrams we can estimate the magnitude of the CM-CR offset using Eq. 2. The diagram we obtain is depicted in the bottom plot of Fig 5. It shows that the residual CM-CR offset changes when the platform is moving. The amount of variation is about $0.010 \mathrm{~mm}$, which agrees with the calculations of Section 2. Thus, we may conclude that structural deformation is the dominating factor limiting the balancing accuracy, which eventually is expected to be around $0.010 \mathrm{~mm}$ in this application. This value is in line with best performance of balancing systems we found documented in the literature which use the batch method. Tab.4. lists several works that report the CM-CR offset they obtained after automatic balancing. 

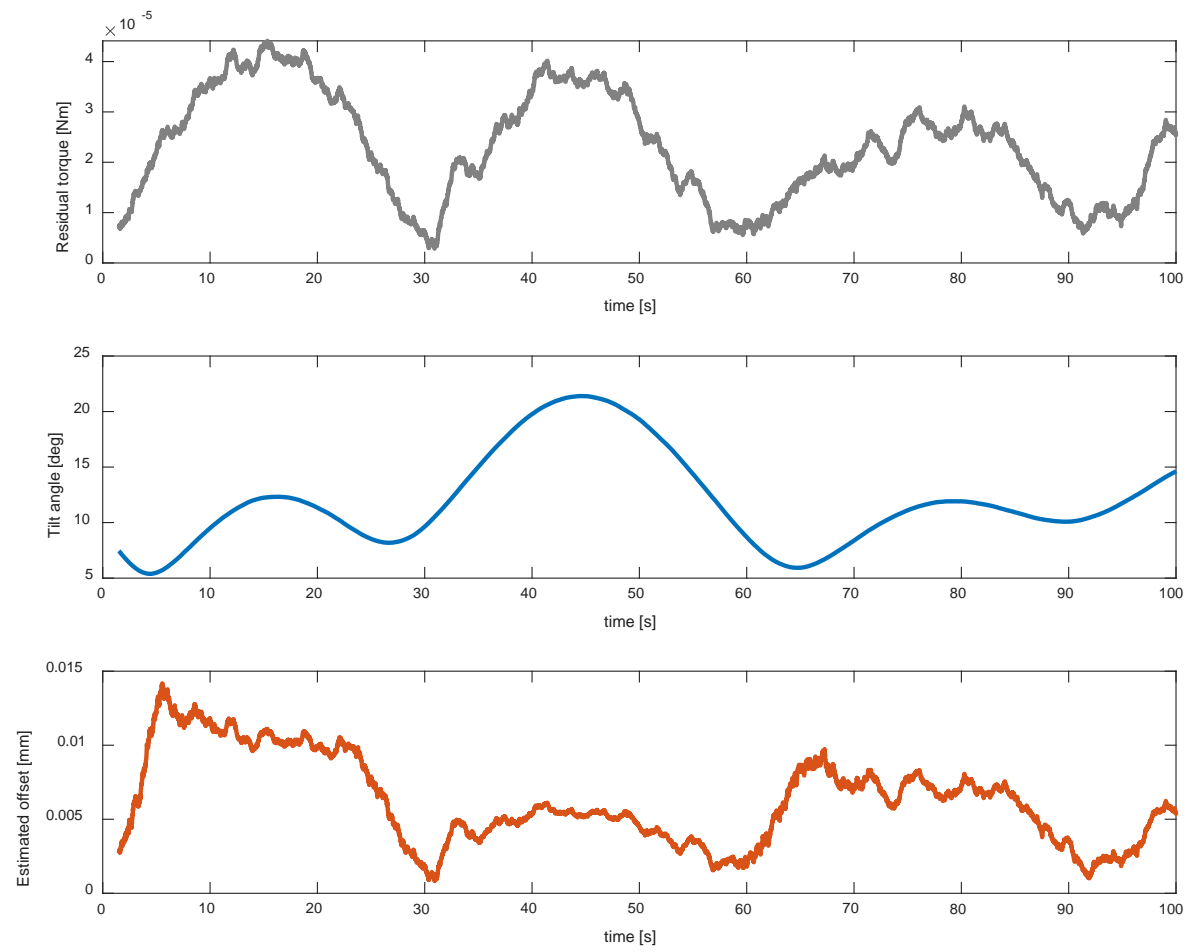

Figure 5. Residual moment (top), tilt angle (middle) and CM-CR offset (bottom) calculated for the system balanced manually.

Table 4 - Comparison of balancing accuracy of systems reported in the literature. The accuracy is measured as the CM-CR offset.

\begin{tabular}{lllll}
\hline Reference & Author(s) & Year & Algorithm & Estimated CM accuracy [mm] \\
\hline$[14]$ & Young & 1998 & Batch & 0.015 \\
{$[16]$} & Prado et al. & 2005 & Batch & 0.025 \\
{$[17]$} & Li and Gao & 2010 & Batch & $<0.200$ \\
{$[18]$} & Liu et al. & 2016 & Batch & $<0.005$ \\
{$[4]$} & Modenini et al. & 2020 & Feedback & $<0.001$ \\
- & Gallina et al. & & Batch & $\sim 0.010$ \\
\hline
\end{tabular}




\section{CONCLUSIONS}

In this work we presented the original design of a floating platform dedicated to testing attitude determination and control system components of small satellites. Conversely to other works documented in the literature, this system features a 3D printed PLA construction. The utilization of plastic reduces the stiffness of the platform and, in turn, the balancing accuracy of the system, which is an important functional parameter of these systems. Nonetheless, we examined the problem and demonstrated that even with these difficulties our design provides balancing accuracy similar to those documented in other solutions in the literature. This result encourages us to experiment also other materials in the future, such as for instance carbonreinforced plastic. This material should increase the system stiffness without losing advantages offered by the 3D printed technology.

\section{REFERENCES}

[1] Small Satellite Market Intelligence Report, Satellite Applications Catapult Limited 2020.

[2] Jung D, Tsiotras P, A 3-DoF Experimental Test-Bed for Integrated Attitude Dynamics and Control Research, AIAA Guidance, Navigation, and Control Conference and Exhibit, 2003.

[3] Chesi S, Perez O, Romano M, A Dynamic, Hardware-in-the-loop Three-Axis Simulator of Spacecraft Attitude Maneuvering with Nanosatellite Dimensions, Journal of Small Satellites, Vol. 4, No. 1, pp. 315-328, 2015.

[4] Modenini D, Bahu A, Curzi G, Togni A, A Dynamic Testbed for Nanosatellites Attitude Verification, Aerospace, Vol. 7 No. 31, 2020.

[5] Kato T, Heidecker A, Dumke M, Theil S, Three-Axis Disturbance-Free Attitude Control Experiment Platform : FACE, Transactions of the Japan Society for Aeronautical and Space Sciences Aerospace Technology Japan, 12 (2013-12).

[6] Varatharajoo R, Ajir R, Ahmad T, Hybrid spacecraft attitude control system, The International Journal of Multiphysics, Vol. 1, No. 2, pp. 221-230, 2007.

[7] Suhadis N, Varatharajoo R, An optimum magnetic control torque generation of a momentum bias satellite, International Journal of Multiphysics, Vol. 8, No. 2, pp. 169180, 2014

[8] Suhadis N, Varatharajoo R, A study of coupled magnetic fields for an optimum torque generation, International Journal of Multiphysics, Vol. 6, No. 1, pp. 73-88, 2012

[9] Micinski P, Bryla J, Martowicz A, Multi-axis Fused Deposition Modeling using parallel manipulator integrated with a Cartesian 3D printer, The International Journal of Multiphysics, Vol . 15, No. 3, pp. 251-263, 2021

[10]Zagorski P, Bangert P, Gallina A, Aerospace Science and Technology, Vol. 66, No. 7, pp. 380-391, 2007.

[11]Wang S, Ma J, Gao S, Balancing Methods on the Three-Axis Air Bearing Platform, Proceedings of the Asia Simulation Conference, 2012.

[12]Chesi S, Gong Q, Pellegrini V, Cristi R, Romano M, Automatic mass balancing of a spacecraft three-axis simulator: Analysis and experimentation, Journal of Guidance, Control, and Dynamics, Vol. 37, No. 1, 2014. 
[13] da Silva R, Guimaraes F, de Loiola J, Borges R, Battistini S, Cappelletti C, Tabletop Testbed for Attitude Determination and Control of Nanosatellites, Journal of Aerospace Engineering, Vol. 32, No. 1, 2019.

[14] Young J, Balancing of a small satellite attitude control simulator on an air bearing, Proceedings of the Utah Space Grant Consortium Symposium, 1998.

[15] Thomas D, Wolosik A, Black J, Cubsat attitude control simulator design, 2018 AIAA Modeling and Simulation Technologies Conference.

[16] Prado Molina J, Bisacchi G, Reyes L, Vicente E, Contreras F, Mesinas M, Three-axis airbearing based platform for small satellites attitude determination and control simulation, Journal of Applied Research and Technology, Vol. 3, No. 3, 2015.

[17] Li Y, Gao Y, Equations of Motion for the Automatic Balancing System of a 3-DOF Spacecraft Attitude Control Simulator, 2010 3rd International Symposium on Systems and Control in Aeronautics and Astronautics.

[18] Liu Y, Li L, Fu Z, Tan J, Automatic Mass Balancing of a Spacecraft Simulator Based on Non-orthogonal Structure, 2016 UKACC 11th International Conference on Control, 2016. 